Adaptive Memory: Generality of the Parent Processing Effect and Effects of Biological Relatedness on Recall

Benjamin M. Seitz ${ }^{1}$, Cody W. Polack ${ }^{2}$, and Ralph R. Miller ${ }^{2}$

${ }^{1}$ University of California, Los Angeles, USA

${ }^{2}$ State University of New York at Binghamton, USA 


\begin{abstract}
The adaptive memory framework posits that the human memory system is an evolved cognitive feature, in which stimuli relevant to fitness are better remembered than neutral stimuli. There is now substantial evidence that processing a neutral stimulus in terms of its relevancy to an imagined ancestral survival scenario produces enhances recall, although there is still disagreement concerning the proximate mechanisms responsible for this effect. Several other mnemonic biases have recently been discovered that similarly appear to reflect evolutionary pressures, including a bias to remember items relevant to an imagined parenting scenario. We tested the generality of this parent processing effect by varying the biological relatedness of the imagined child. We also varied the biological relatedness of a child during an imagined thirdperson survival processing scenario. Across four experiments, we found evidence that simply altering the described biological relatedness of a child in the parenting scenario and third-person survival processing scenario can affect recall, such that items are better remembered when made relevant to a biological child compared to an adopted child. How these findings inform the general adaptive memory framework is discussed.
\end{abstract}

Key words: adaptive memory; survival processing effect; reproduction processing effect. 


\section{Adaptive Memory: Generality of the Parent Processing Effect and Effects of Biological Relatedness on Recall}

The adaptive memory framework posits that biological memory systems, including those of humans, are the product of millions of years of natural selection; thus, evidence of these selective pressures should be observable in mnemonic performance under conditions relevant to evolutionary fitness. The first report providing support for this hypothesis was Nairne, Thompson, and Pandeirada (2007). Combining their view with a levels-of-processing approach (Craik \& Lockhart, 1972), Nairne et al. had participants encode neutral concrete nouns based on their relevancy to an imagined scenario about surviving in an ancestral grassland environment. Recall for words encoded in this manner was far superior to that for the same words encoded for their relevancy to an imagined scenario that involved moving to a foreign land. In fact, encoding items in this manner yields better recall than nearly all other encoding techniques examined to date (Nairne, Pandeirada, \& Thompson, 2008). This enhancement of recall is known as the 'survival processing effect.' Over the past decade, a large number of successful replications of this effect have been reported and a recent meta-analysis including 90 experiments yielded effect sizes of around $\eta_{p}{ }^{2}=.06-.09$ for between-subject designs and $\eta_{p}{ }^{2}=$ .15 - .18 for within-subject designs (Scofield, Buchanan, \& Kostic, 2018).

Since the initial 2007 publication on survival processing, a number of researchers have documented mnemonic biases to the human memory system that appear to reflect evolutionary pressures. For example, concrete items (i.e., desk, chair) are better remembered if they are described as having been touched by someone who has a contagious disease rather than by a healthy individual (Bonin, Thiebaut, Witt, \& Méot, 2019; Fernandes, Pandeirada, Soares, \& Nairne, 2017). Additionally, after showing faces of the people who had touched the items, participants demonstrated a better memory for items paired with faces that appeared sick than items paired with pictures of faces that appeared healthy. Source memory is also increased for potentially contaminated items (Bonin et al., 2019). These findings suggest the memory system 
preferentially remembers potential sources of contamination. Hou and Liu (2019) found that compared to memory of neutral faces, there was enhanced memory for faces perceived as trustworthy or untrustworthy during a scenario involving survival. This is consistent with other findings that demonstrate a source memory bias for the faces of people who are described as cheaters (Bell \& Buchner, 2012). Using a novel procedure, New, Krasnow, Truxaw, and Gaulin, (2007) had participants walk through a farmer's market and then recall the locations of various vendors. They found better memory for the locations of stands that sold items with higher caloric density. Moreover, in studying memory for wordlists, a bias for animate versus inanimate objects has been consistently observed, even while controlling for aspects like imaginability, meaningfulness, saliency, valence (Nairne, VanArsdall, \& Cogdill, 2017; Nairne, VanArsdall, Pandeirada, Cogdill, \& LeBreton, 2013).

A number of these mnemonic biases also appear to be related to reproduction. For instance, Seitz, Polack, and Miller (2018) found that processing items based on their relevancy to an imagined parenting scenario, resulted in a memory benefit for those items comparable to that of the survival processing effect. This memory bias may be one of a suite of cognitive and behavioral adaptations that comprise the parental care motivational system, that were presumably selected for to aid our species in raising offspring (Schaller, 2018, 2020). Whether or not a memory benefit exists for selecting a mate appears to be contingent on the experimental procedure. Those that have created novel encoding scenarios similar to that of survival processing but with the emphasis on finding a mate have not found significant memory benefits (Derringer, Scofield, \& Kostic, 2017; Klein, 2013; Sandry, Trafimow, Marks, \& Rice, 2013; Seitz et al., 2018). However, there is evidence that memory can be influenced by aspects of human mating outside of the survival processing paradigm. For example, Pandeirada, Fernandes, Vasconcelos, and Nairne (2017) found enhanced memory for the descriptions of individuals being evaluated as potential long-term mates compared to those same descriptions if the individual is being evaluated as a potential coworker. Baker, Nicole Sloan, Hall, Leo, and 
Maner (2015) found increased recall for details of a story when men were also viewing images of attractive faces of the opposite sex compared to average faces of the opposite sex and that these effects were not driven by changes in arousal or mood. Likewise for female participants, Smith, Jones, Feinberg, and Allan (2012) reported enhanced visual object memory for items that were accompanied by a masculinized male voice during the encoding phase, compared to the same items being accompanied by a feminized male voice. Additionally, female undergraduates who watched a short video of a man introducing himself remembered more about his physical features than the verbal statements about himself if they had been first told to consider the man as a short-term mate. Meanwhile, if the female participants were told to consider the man as a potential long-term mate before watching the video, they better remembered his verbal statements than his physical appearance (Horgan, Broadbent, McKibbin, \& Duehring, 2016). Thus, by placing the emphasis on parenting a child, or by using different procedures than that of the survival processing effect, there appears considerable evidence of mnemonic biases towards information relevant to reproductive fitness.

The Omega Scaling Factor

The pattern that emerges from the reviewed literature is that the perceived fitness relevance of encoded information appears to potentiate its being remembered. Similar arguments have been made in the domain of learning theory, as much work has demonstrated the differential occurrence of learning as a consequence of using biologically significant stimuli and outcomes (Denniston, Miller, \& Matute, 1996; Domjan \& Krause, 2017; Garcia \& Koelling, 1966; Seligman, 1970; Shettleworth, 1972). To account for these mnemonic biases, Seitz, Blaisdell, Polack, and Miller (in press) proposed a computational parameter, Omega, that quantifies the perceived fitness relevance of information. Thus, this value can be used to scale the predictions of other computational models of learning and memory, to account for differences in the memorability of fitness relevant compared to fitness-irrelevant information. 
This parameter predicts a positive correlation between the perceived fitness relevance of information and the likelihood of that information being remembered.

In the present study, we attempted to both replicate our parent processing effect and to test the efficacy of the proposed Omega scaling factor. Toward that end, we created several new processing scenarios that differed with respect to raising one's own biological child, an adopted child, or raising of a pet dog, and compared these to the original survival scenario and a pleasantness control group. We henceforth refer to our original parenting scenario (Seitz et al. 2018) as the biological parenting scenario. Based on this encoding scenario, we have generated four scenarios that putatively differ in terms of perceived fitness value, with one's personal survival being the most relevant to evolutionary fitness and the raising of a pet dog being the least relevant to evolutionary fitness. We predict better memory for items processed based on their relevancy to one's own survival and the parenting of their biological child, compared to the parenting of an adopted child or raising of a pet dog.

\section{Experiment 1}

Experiment 1 sought to (a) replicate the parent processing effect established by Seitz et. al. (2018), and (b) explore the generality of this effect to others beyond one's own child, as a direct test of our Omega scaling factor. Experiment 1 and all other experiments reported here were approved by SUNY-Binghamton and/or the University of California, Los Angeles Institutional Review Boards.

\section{Method}

Participants: A total of 250 (176 female) SUNY-Binghamton undergraduates volunteered to take part in this study to fulfill part of a course requirement. The number of participants reflects estimated power based on the original survival processing study (Nairne et al., 2007) as well as a number of studies conducted in our laboratory, and was selected to provide approximately $90 \%$ power to detect a medium $(f=0.25)$ effect (Faul, Erdfelder, Lang, \& Buchner, 2007). 
Materials: Stimuli were presented on computer screens in individual cubicles and sessions lasted less than 30 minutes. Type of scenario was manipulated across participants and the scenario descriptions (see Table 1) were shown to participants prior to presenting them with a common list of 45 unrelated concrete nouns. Of these 45 words, 35 had been used in Experiment 2b of Seitz et al. (2018). That study had also included 45 words and 10 of those 45 words were excluded because on average they were rated as more relevant in the Survival scenario than in the Parenting scenario. We replaced the 10 removed words with 10 more nouns from Nairne et al. (2007). Our entire set of 45 words were drawn from Nairne et al. (2007), with words that were implausible in an ancestral landscape purposefully excluded. Following exposure to their respective scenarios, participants were asked to rate the 45 nouns in terms of how relevant they were to the scenario to which the participant was assigned (with the exception of the Pleasantness scenario, in which the perceived pleasantness of the word was rated). All 45 words were presented in the same fixed random order. The ratings and recall data for the first 5 words rated were discarded, being treated as practice words in all scenarios. The 40 remaining words that were critically assessed for recall are listed in Table 2.

In light of the ultimate goal of reproduction being to produce viable offspring who carry one's alleles, our Biological Parenting condition asked participants to rate words based on their relevancy to raising their own hypothetical baby in an ancestral environment. This scenario follows a schematic structure similar to that from the original survival processing scenario and we used the same wording for this condition in this experiment as was used in Experiment $2 b$ of Seitz et al. (2018, referred to there simply as the Parenting scenario).

The Adopted Parenting condition examined whether a parent processing effect would occur when the child in question is not biologically related to the participant. As such, the described scenario should be of lesser perceived fitness relevance to participants and, according to our Omega scaling factor, result in poorer recall. The phrasing of the Adopted 
Parenting scenario was modeled after the structure of the original parenting scenario and modified accordingly (see Table 1).

The Pet-raising condition also explored the generality of the parent processing effect by varying the fitness relevance of the scenario. In this scenario the participant is told to imagine they have domesticated a dog and are asked to imagine raising it, nourishing it, and providing care for it. Once again, the phrasing of the Pet-raising scenario followed the structure of the Biological Parenting scenario. The last two conditions were the original Survival Processing scenario and the Pleasantness scenario that were used throughout Seitz et al. (2018) and were nearly identical to the phrasing of Nairne et al. (2007) for those conditions. Table 1 provides a full list of the scenario descriptions.

Procedure: Participants were asked to read and sign an informed consent form and enter their age and gender on a keyboard. Participants were then presented with the scenario to which they had been randomly assigned for 30 s on-screen. They were then prompted to press the space bar to advance to the experiment. Following presentation of the assigned scenario, they were next asked to rate how relevant (or pleasant) each of the 45 words was on a 5-point scale ranging from 1 (very irrelevant [very unpleasant]) to 5 (very relevant [very pleasant]). This was accomplished by sequentially presenting each of the words for $5 \mathrm{~s}$ in a random order that was the same for all participants. The rating of each word needed to occur within the 5-s presentation; otherwise the word disappeared and no rating was recorded. Stimuli remained onscreen for the full $5 \mathrm{~s}$, regardless of whether a rating had been entered. The intertrial interval from offset of one word to onset of the next word was $250 \mathrm{~ms}$. Upon completing the ratings of all items, participants performed a distraction task (simple algebraic problems with paper and pencil) for $2 \mathrm{~min}$. After this, the screen flashed red and white for $10 \mathrm{~s}$ to orient participants back to the next instruction screen, which asked them to perform a free recall test on all 45 of the previously rated words. Participants were given $10 \mathrm{~min}$ to write as many recalled words as possible on a piece of lined paper. Participants were asked to stay for the entirety of the 10-min 
recall period, after which they were thanked for their participation and debriefed. Two research assistants independently scored all of the completed recall sheets. Each assistant selected items from the presented list that they thought best matched the participants' responses. Responses were considered valid only if both assistants chose the same word. The valid words were then recorded along with the participants' relevance or pleasantness ratings for all of the words except the first five words that had been presented during the relevancy rating (i.e., encoding) phase which were regarded as warm up items.

\section{Results and Discussion}

The threshold for significance of all statistical tests was set at $p<.05$. Ninety-eight percent of all presented words were rated within the 5-s presentation time, and the number of unrated words did not differ significantly across groups. To avoid selection biases and because no condition produced a significantly higher or lower number of unrated words than other conditions, the recall data described below was in no way constrained.

Figure 1a summarizes the results of greatest interest to our research question; that is, it presents proportions of correctly recalled words (out of 40) within each experimental condition (i.e., scenario type). A one-way analysis of variance (ANOVA) revealed a main effect of condition, $F(4,240)=7.41, p<.001, \eta_{p}{ }^{2}=.110$. As predicted by the Omega scaling factor, recallability of neutral items appeared to decline as the biological relatedness to oneself decreased (personal survival $>$ raising biological child $>$ raising adopted child $>$ raising adopted pet). Unfortunately, analysis of mean relevancy rating (see Figure $1 \mathrm{~b}$ ) revealed a strong main effect of condition, $F(4,245)=7.41, p<.001, \eta_{p}{ }^{2}=.108$, which undermined the usefulness of a simple analysis of differences in recall proportion between conditions, due to possible congruity effects (Butler, Kang, \& Roediger, 2009).

Seitz et al. (2018), however, developed a method to analyze recall data when differences in relevancy ratings are observed. This consists of calculating the proportion of correctly recalled words for each experimental condition at each individual relevancy rating 
level. Simply put, for each participant we counted how many words were rated as a 1, 2, 3, 4, or 5 for relevancy to the participant's scenario, and then computed the proportion of those words that were correctly recalled at each rating level by that participant. Mean item relevancy rating per condition were used in those rare instances when participants had not assigned a relevance rating to a given item. This was chosen because our analyses were primarily concerned with differences between conditions. Figure 1c depicts the mean proportion of words recalled correctly at each rating level. A 5 (conditions) x 5 (rating levels) mixed measures ANOVA was performed on proportion of items recalled. It revealed a main effect of condition, $F(4,245)=$ 4.21, $p=.003, \eta_{p}^{2}=.064$, and a main effect of rating, $F(4,980)=32.61, p<.001, \eta_{p}^{2}=.078$. The strong effect of relevancy rating demonstrates the importance of this kind of analysis when there are overall differences in relevancy ratings across scenarios because item rating is significantly correlated with its being recalled. Unfortunately, this same analysis also revealed a significant interaction between rating and recall, $F(16,980)=1.84, p=.022, \eta_{p}{ }^{2}=.018$, which creates difficulty in interpreting the difference in proportion of words recalled across experimental conditions. The interaction was likely due to congruity effects that differentially impacted recall by the different conditions at relevancy levels at which the greatest differences in number of words rated at those levels occurred, that is, rating levels 1 and 5 .

To provide an analysis which would be less influenced by this interaction, we discarded the data from extremely relevant (rated 5) and extremely irrelevant (rated 1) words (see the anchored parts of Figure 3). By doing so, we were able to analyze the proportion of words recalled for each scenario as a function of relevancy rating (2, 3, and 4 only), thereby avoiding rating levels at which relevancy ratings differed most across conditions. A mixed measures 5 (conditions) $\times 3$ (rating levels) ANOVA was conducted on the proportion of words correctly recalled from words ranked 2-4 in relevancy. This revealed a main effect of condition, $F(4,245)$ $=4.29, p=.002, \eta_{p}^{2}=.065$, and an effect of relevancy rating, $F(2,490)=14.36, p<.001, \eta_{p}{ }^{2}=$ 
.029. Additionally and critically, no significant interaction between ratings and recall was found, $F(8,490)=1.06, p=0.39$. Planned comparisons revealed that the proportion of words recalled from those rated 2-4 in relevancy (pooled across ratings of 2, 3, and 4 ) in the Survival scenario resulted in significantly higher recall than the Pleasantness scenario, the Pet-raising scenario, and the Adopted Parenting scenario, all ps $<0.01$. Replicating the results of Seitz et al., (2018), no difference in recall was found between the Survival and Biological Parenting scenario, $t(298)$ $<1.0$, and words rated in the Biological Parenting scenario were recalled significantly better than the same words rated in the Pleasantness scenario, $t(298)=2.37, p=0.02$. Of central interest, words rated in the Biological Parenting scenario were recalled at a significantly higher rate than those rated in the Adopted Parenting scenario, $t(298)=2.10, p=.038$. This suggests that the parent processing effect was stronger for one's own biological offspring than for adopted children. There was no difference between adopted parenting and pet raising, $t(298)<$ 1.0 .

Experiment 1 thus replicated the well-established survival processing effect as well as the recently discovered (biological) parent processing effect. Our data also suggest that the parent processing effect is stronger when the imagined child in question is biologically related to the participant. It is rather remarkable that simply changing the wording from 'parented a baby' to 'came across a baby that is not yours' could have such an impact on mnemonic performance. That said, this finding lends itself nicely to the view that evolved human memory system is enhanced on fitness related tasks, as has been suggested by many researchers (e.g., Anderson \& Schooler, 2000; Barkow, Cosmides, \& Tooby, 1992; Nairne \& Pandeirada, 2016).

Furthermore, this finding is also in line with the proposed Omega scaling factor, whereby the perceived fitness relevance of information is positively associated with its likelihood of being remembered.

\section{Experiment 2}


In the previous experiment, we found evidence of a difference in recall for items processed based on their relevance to an imagined biological parenting scenario compared to an imagined adopted parenting scenario. In this experiment, we sought to replicate this finding and to examine how biological relatedness affects memory in third-person survival processing. Third-person survival processing effects were initially demonstrated during inquiries into whether the survival processing effect could be explained by self-referential processing. Kang, McDermott, and Cohen (2008) had participants rate words for their relevancy to the survival of somebody other than the participant. Specifically, participants watched a short clip about a man stranded on an island or a man who was planning to rob a bank and were then told, "Your task is to rate how relevant each of these words might be for the character in the video clip in terms of planning for his survival (or planning his bank heist)." The planning of a bank heist is a common comparison scenario to test the effectiveness of the survival processing scenario that was first introduced in Experiment 1 of Kang et al. (2008). They found enhanced recall for the third-person survival scenario compared to the third-person bank robbing scenario, suggesting self-referential encoding is not an essential factor in producing the survival processing effect. Furthermore, Weinstein, Bugg, and Roediger (2008) found no difference in recall performance when comparing the original survival scenario to the survival of one's friend. The authors concluded that the survival processing effect cannot fully be explained by self-referential processing because the mnemonic advantage extends to processing words when thinking about the survival of others.

Related to these observations, Cunningham, Brady-Van den Bos, Gill, and Turk (2013) created a similar scenario that replaced "friend" with "Prime Minister David Cameron" and found the mnemonic advantage did not extend to this new third person. Thus, there appears to be a difference in the processing of information concerning a third person who is a friend compared to a third person who is a known stranger, which might be a reflection of the importance of caring for kin (Kazanas \& Altarriba, 2015). In fact, this finding (though not specifically the 
evolutionary interpretation) is supported by older work demonstrating the role of intimacy between the participant and an imagined third person and recall (Bower \& Gilligan, 1979).They found similar rates of recall for items related to oneself compared to related to one's mother, but worse recall for items related to a stranger (i.e., "Walter Cronkite"). More recently, Kole and Healy (2011) found that information describing a hypothetical identical twin was better remembered than information describing a first cousin. All of these findings are well explained by the proposed Omega scaling factor, but of course, these are post-hoc explanations.

Thus in this experiment, we examined the effect of biological relatedness (biological and adopted child) on two different imagined tasks (parenting and third person survival processing) using a $2 \times 2$ design. In addition to these four conditions, we also included a pleasantness control condition to provide a widely used baseline against which the effectiveness of the four experimental scenarios could be assessed.

\section{Method}

Participants. A total of 421 (266 females) SUNY-Binghamton undergraduates volunteered to take part in this study to fulfill part of a course requirement. The increased sample size was informed by power analyses based on the experimental design and the results obtained from Experiment 1. Unfortunately, time constraints on running participants resulted in our ending short of our goal of 100 participants per each experimental condition (and 50 for the pleasantness condition). Our conditions thus contained around $94+/-5$ participants each (Biological Parenting = 97, Adopted Parenting = 94, Adopted Third-person Survival = 89, Biological Third-person Survival $=96$, Pleasantness $=45$ participants), due to the nature of random assignment. Given that inferential statistics do not require equal group sizes and our reaching $94 \%$ of our target sample size, we conducted our analyses using these data.

Materials. To reduce the likelihood of differences in relevancy ratings between conditions, we conducted a pilot study $(\mathrm{N}=60)$ in which 70 words were presented to participants in either the adopted parenting scenario or the biological third-person survival 
scenario. We reasoned that items would be rated similarly regardless of biological relatedness and thus our main objective was to make sure relevancy ratings would be similar for the two different tasks (i.e., parenting and third-person survival processing). We then eliminated the 35 words with the largest mean differences in relevancy ratings between the two tasks, leaving us with 35 words. The ratings and recall data of the first 5 words (the same for all participants) were discarded, being treated as practice words in all scenarios (see Table 3 for the 30 words used for analyses).

The adopted parenting, biological parenting, and pleasantness scenarios were taken verbatim from Experiment 1. Two new scenarios were created for this experiment (see Table 1 for full scenario descriptions; Experiment 4 below additionally assessed how these scenarios compare to others on various descriptive characteristics). Both of the new scenarios asked participants to imagine a baby surviving in the grasslands without mention of the participant's being involved in the survival of the baby, with the crucial difference being whether or not it was your baby or a baby that is not yours.

Procedure. The procedure used in Experiment 2 was the same as that used in Experiment 1.

\section{Results and Discussion}

A one-way ANOVA on proportion of words recalled revealed a significant main effect of Condition, $F(4,416)=3.90, p=0.004, \eta_{p}^{2}=.036$ (see Figure 2a). To gauge the effectiveness of these encoding scenarios, two of which were entirely novel, we compared recall performance from each group to the recall performance of words rated on their perceived pleasantness, which is an encoding scenario known to elicit moderately strong mnemonic performance. Planned comparisons revealed the two scenarios describing a biological child resulted in recall that was significantly better than in the pleasantness condition $p s<0.05$, but that the adopted third-person processing scenario was only marginally significant relative to pleasantness, $t(132)$ 
$=1.91, p=0.058$, and the adopted parenting scenario was not any different than pleasantness, $t(137)<1.0$. Thus, we replicated our biological parent processing once more and also demonstrated enhanced memory resulting from third-person survival processing of a biological child relative to a pleasantness control group.

Of greatest interest to this experiment, we conducted a 2 (Relatedness) $\times 2$ (Task) ANOVA on proportion of words recalled which revealed a small but significant, main effect of relatedness, $F(1,372)=4.87, p=.028, \eta_{p}{ }^{2}=.013$, and a small but significant, main effect of task, $F(1,372)=4.80, p=.029, \eta_{p}^{2}=.013$, but no interaction between the two, $F(1,372)<1.0$ (see Figure 2b). Unfortunately, despite our large pilot study to select words with the smallest differences in relevancy ratings across scenarios, a 2 (Relatedness) $\times 2$ (Task) ANOVA of Rating data revealed a marginally significant effect of relatedness $F(1,372)=2.98, p=.085, \eta_{p}{ }^{2}$ $=.085$, and a significant effect of task, $F(1,372)=9.79, p=.002, \eta_{p}{ }^{2}=.002$, but no interaction between relatedness and task, $F(1,372)<1.0$ ( see Figure $2 c$ ). Thus, to provide a more meaningful analysis that controlled for congruity effects, we conducted a 2 (Relatedness) $\times 2$ (Task) x 5 (Rating Proportions) mixed ANOVA which revealed a marginally significant main effect of relatedness, $F(1,372)=3.71, p=.055, \eta_{p}^{2}=.010$, no main effect of task, $F(1,372)<$ 1.0 , a very strong effect of Rating, $F(4,1488)=78.12, p<.001, \eta_{p}{ }^{2}=.12$, and a marginally significant interaction between relatedness and task, $F(1,372)=3.35, p=.068, \eta_{p}^{2}=.009$. (see Figure $2 \mathrm{~d}$ ). No interaction was found between rating and task, $F(4,1488)=1.17, p=0.32$, or between rating and relatedness, $F(4,1488)=1.38, p=.24$, nor was there a significant threeway interaction, $F(4,1488)<1.0$. Planned comparisons tested the difference between adopted and biological third person survival processing and found a significant difference in recall, $t(923)$ $=3.27, p<0.01$, but there was no difference between biological parenting and adopted parenting, $t(953)<1.0$. Thus, biological relatedness again appears to affect recall, although here, the difference was observed only for the third-person survival processing task, not the parenting task as had been observed in Experiment 1. 
Experiments 1 and 2 provided inconsistent evidence for an effect of biological

relatedness on the parent processing effect. In Experiment 1, planned comparisons between the two scenarios revealed a significant difference with memory for items processed in terms of their relevancy to parenting a biologically related child being remembered better than when processed in terms of relevancy to parenting an adopted child, but in Experiment 2, after adjusting for differences in relevancy ratings, this effect was absent. However, in Experiment 2 there was evidence of biological relatedness affecting recall when items were processed in terms of their relevancy to the survival of a third person. Thus, there exists evidence suggesting that biological relatedness can substantially impact mnemonic performance, as predicted by the Omega scaling factor. To explore this further, specifically within the parent processing scenario, we conducted another experiment with the same two conditions as Experiments 1 and 2 to better establish whether a difference between biological and adopted parenting actually exists.

\section{Experiment 3}

Given the uncertainty of the impact of biological relatedness on recall in the parenting scenario, we conducted a simple between-subjects study to further assess the influence of biological relatedness. Additionally, we conducted an internal meta-analysis, including data from all participants in all three experiments run in either the adopted or biological parenting scenario, to assess the size of this potential effect.

\section{Methods}

Participants. A total of 43 (21 female) SUNY-Binghamton and 57 UCLA (37 female) undergraduates volunteered to take part in this study to fulfill part of a course requirement $(\mathrm{N}=$ 100). 50 participants per condition was chosen for consistency with previous experiments of this sort. Participants from both universities were randomly assigned to each condition and the computer programs were identical.

Materials. Using the rating data from Experiment 1, we selected 35 words with the smallest mean differences in relevancy ratings to reduce the possibility of differential relevancy 
ratings between the two conditions. The two parenting scenarios, adopted parenting and biological parenting, were identical to those used in Experiments 1 and 2.

Procedure. The procedure used in Experiment 3 was the same as that used in Experiments 1 and 2.

\section{Results and Discussion}

An independent samples t-test revealed no difference in mean relevancy ratings between the biological parenting condition $(M=3.03, S D=.43)$ and adopted parenting scenario $(M=3.03, S D=.44), t(98)<1.0$. Additionally, no significant difference was found in mean proportion of items recalled between the biological parenting condition $(M=0.61, S D=.16)$ and adopted parenting condition $(M=0.58, S D=.12), t(98)=1.07, p=.30$. Thus, we have presented three experiments that have either directly or indirectly assessed differences in recall performance of adopted and biological parenting processing scenarios. Despite recall performance in the biological parenting condition outperforming recall performance in the adopted parenting scenario in each of these experiments, only in Experiment 1 was this difference statistically significant. Thus, to better gauge the size of this possible effect, we conducted a 'meta-analysis' on the recall and rating data from all of the participants in all three experiments who had served in either of these two conditions.

This analysis included 197 participants in the biological parenting scenario and 194 in the adopted parenting scenario. The mean relevancy rating given to items in the biological parenting scenario $(M=3.18, S D=.51)$ did not significantly differ from the ratings of items in the adopted parenting scenario $(M=3.11, S D=.56), t(389)=-1.25, p=.21$. However, there was a significant difference in proportion of items recalled between the two scenarios, such that items processed in the biological parenting scenario $(M=.51, S D=.15)$ were better recalled than items in the adopted parenting scenario $(M=.48, S D=.15), t(389)=-2.1, p=.036$, Cohen's $d=$ .21 (see Figure 3). Therefore, we conclude that biological relatedness seems to impact recall performance within the parent processing scenario, but this is only a small effect. 


\section{Experiment 4}

The studies reported above consist of novel encoding scenarios that were generated for the purposes of testing our specific hypotheses. Thus, given the design of these experiments, there is reason to ask whether these scenarios differ appreciably on a variety of descriptive qualities that could in principle be responsible for the observed differences in recall. To that end, we asked a new group of participants to rate our scenarios on how "interesting," "imaginable," "arousing," "familiar," "aversive," and "challenging" they considered these scenarios to be. These descriptive qualities were used previously by Sandry et al. (2013) when generating novel encoding scenarios, and we reported them in Seitz et al., (2018, Experiment 1b) for our novel scenarios including the biological parenting scenario and the original survival processing scenario used throughout these studies. The descriptive qualities for biological parenting and survival processing are re-reported here for ease of comparison, as well as these qualities for the pet raising scenario, adopted parenting scenario, biological third-person processing scenario, and adopted third-person processing scenario.

\section{Methods}

Participants. Sixty SUNY-Binghamton undergraduates (34 female; age range 18 - 26) volunteered to take part in this study to fulfill part of a course requirement. Participants who had participated in the preceding studies were excluded from participating in this study.

Materials. Ten unique scenarios were each presented once to each participant. Presentation order of the 10 scenarios was partially counterbalanced using a Latin square. Additionally, the scenarios were systematically separated so that scenarios we deemed to be similar were never presented in immediate sequence. That is, we identified clusters of scenarios that were so similar that exposure to one might influence the ratings of the next if they were presented one right after the other. Although ratings for 10 scenarios were measured within this single experiment, we report on only six of these scenarios here because the other four 
scenarios were not used in the present experiments; however, they are reported in Seitz et al. (2018). Six questions were asked of each participant for each scenario. The six questions ("interesting," "imaginable," “arousing," "familiar," "aversive," and "challenging”) were asked in the same order for all participants (e.g., "How interesting would you consider this scenario?"). The 1-5 point Likert scale used for the ratings was displayed horizontally, and participants were asked to make a rating using the keyboard.

Procedure. See Seitz et al. (2018, Experiment 1b) for a full description of the procedure. Participants were randomly assigned to one of the ten conditions that collectively counterbalanced scenario order, and each condition was completed by a total of six participants. The same six questions were asked for all scenarios. No words were rated for relevancy and there was no subsequent memory test.

\section{Results and Discussion}

The mean ratings of the descriptive qualities of each scenario are depicted in Table 4. Of central interest is whether there were any differences between the descriptive qualities of the biological and adopted parenting scenarios and also between the descriptive qualities of the biological and adopted third-person survival processing scenarios, but we were also interested in how all of the scenarios used throughout these studies differed from one another. Thus, we conducted one-way ANOVAs for the responses to each question, using condition as the main factor. Given the large number of potential analyses and our lack of predictions concerning differences, we used Tukey HSD post-hoc comparisons following any significant effects of condition for each question.

\section{Arousing}

In measuring differences in how arousing participants found the scenarios to be, a oneway ANOVA revealed no significant main effect of scenario, $F(5,354)=1.20, p=0.31, \eta_{p}{ }^{2}=$ .017 .

\section{Aversive}


In measuring differences in how aversive participants found the scenarios to be, a oneway ANOVA revealed a significant main effect of scenario, $F(5,354)=3.06, p=0.049, \eta_{p}{ }^{2}=$ .031. A Tukey HSD comparison found the dog raising scenario to be significantly less aversive than the third-person survival of a biological child scenario, $t(118)=-2.98, p=0.036$. There were no other significant differences, and it is unsurprising that the most aversive scenario was imagining one's own child stranded in the grasslands in need of survival.

\section{Challenging}

In measuring differences in how challenging participants found the scenarios to be, a one-way ANOVA revealed a significant main effect of scenario, $F(5,354)=9.75, p<0.001, \eta_{p}{ }^{2}=$ .121. Tukey HSD comparisons found the dog raising scenario to be significantly less challenging than the five other conditions, all $p s<0.001$. There were no other significant differences between the scenarios.

\section{Familiar}

In measuring differences in how familiar participants found the scenarios to be, a oneway ANOVA revealed a significant main effect of scenario, $F(5,354)=6.52, p<0.001, \eta_{p}{ }^{2}=$ .084. Tukey HSD comparisons found the dog raising scenario to be significantly more familiar than the five other conditions, all $p s<0.05$. but again, there were no other significant differences between the scenarios.

Imaginable

In measuring differences in how imaginable participants found the scenarios to be, a one-way ANOVA revealed a significant main effect of scenario, $F(5,354)=8.18, p<0.001, \eta_{p}{ }^{2}=$ .104. Tukey HSD comparisons revealed the dog raising scenario to be more imaginable than the adopted parenting, third-person survival of an adopted child, and third-person survival of a biological child, all $p s<0.05$. Additionally, the biological parenting scenario was rated as more imaginable than the third-person survival of a biological child, $t(118)=3.20, p=0.019$. The 
survival scenario was also more imaginable than the third-person survival of a biological child, $t(118)=4.19, p<0.001$

Interesting

In measuring differences in how interesting participants found the scenarios to be, a one-way ANOVA revealed no significant main effect of scenario, $F(5,354)=1.58, p=0.17, \eta_{p}{ }^{2}$ $=.022$.

Taken together, there appear to be no clear pattern of descriptive qualities about the encoding scenarios that can account for the differences in recall observed throughout these experiments. Therefore, we suggest the differences in recall are likely reflective of factors related to how the tasks differ on evolutionary fitness as opposed to more trivial differences in the descriptive qualities of the scenarios themselves.

\section{General Discussion}

Over the past decade following Nairne et al.'s (2007) publication reporting on the survival processing effect, a large number of other mnemonic biases that appear to reflect selective pressures have been discovered. These involve biases with respect to remembering potential sources of contamination (Bonin et al., 2019; Fernandes et al., 2017; Gretz \& Huff, 2019), remembering animate versus inanimate objects (Gelin, Bonin, Méot, \& Bugaiska, 2018; Nairne, VanArsdall, \& Cogdill, 2017; Nairne, VanArsdall, Pandeirada, Cogdill, \& LeBreton, 2013), remembering suspicious characters (Hou \& Liu, 2019), and remembering items relevant to raising ones child (Seitz et al., 2018) or finding a mate (Pandeirada et al., 2017; for a review, see Seitz et al., in press). These findings are suggestive of general evolutionary constraints to memory, and Seitz et al. (in press) suggested there exists a positive relationship between the perceived fitness value of information and its likelihood of being remembered. This can be modeled using the Omega parameter. To test this parameter, we created several novel scenarios that differed with respect to the evolutionary fitness involved in their described tasks. 
The tasks were primarily to either imagine oneself raising the child as described by Seitz et al., (2018), or to imagine the child surviving in the grasslands, which is similar to scenarios used by Cunningham et al., (2013) and Weinstein et al., (2008).

In Experiment 1, we created two novel scenarios, an adopted parenting scenario and a pet-raising scenario. We found that recall of items consistently decreased as the biological relatedness of the participant decreased with respect to the main character within the scenario from the participants themselves, to one's own biological child, to an adopted child, to a pet dog (see Figure 1a). It is impressive that such small differences in the phrasing of encoding scenarios could elicit these noticeable differences in recall performance, and that the observed recall patterns follow the predictions of our Omega scaling factor. After controlling for differences in item relevancy ratings, we saw a small but statistically significant difference in recall for words processed in the biological parenting scenario compared to the adopted parenting scenario, and we also replicated our previously established biological parenting advantage compared to a pleasantness control group.

Experiment 2 sought to replicate this difference between biological and adopted parenting and explore the underlying difference using a slightly different task. We manipulated both the task (parenting a child vs. explicit survival of the child) and biological relatedness (biological vs. adopted) of the child to create four scenarios and increased the power of our study to detect potential differences. We found that scenarios related a to a biological child produced better recall than a pleasantness control group. But the adopted third-person survival scenario was only marginally significant compared to pleasantness and the adopted parenting scenario was not statistically different than pleasantness condition. Rating words based on their perceived pleasantness has long been used as a baseline standard for encoding scenarios (Nairne \& Pandeirada, 2008). After controlling for potential congruity effects due to differences in mean item ratings in Experiment 2, we found that the biological relatedness of the child 
affected recall for the third-person survival processing task, but not the parenting task, despite what had been observed in Experiment 1.

Given these differences in the effect of biological relatedness to parent processing, in Experiment 3 a simple study pitted biological and adopted parenting against each other, taking care to eliminate potential differences in item relevancy ratings between the two scenarios. While words were once again better recalled in the biological parenting scenario compared to the adopted parenting scenario, the difference was not statistically significant. That said, given this consistent pattern of biological parenting resulting in better recall than adopted parenting in all of our experiments, an meta-analysis was conducted across Experiments 1-3 and found a small but significant difference between the two conditions, Cohen's $d=.21$. Thus, we conclude that biological relatedness can affect both third-person survival processing and parent processing, although these are admittedly small effects. Interestingly, a recent report from Krause et al. (2019) similarly tested how the inclusive fitness of others during a survival processing scenario impacted memory. They observed no differences in memory performance when processing words based on their relevancy to the survival of, "you and your family (including relatives and your own children)" compared to, "you and several unrelated people with whom you live with." While interpreting null results can be difficult, this result may be due to the fact that these scenarios included reference to the participant including themselves in the scenario and thus may have prompted participants to primarily imagine their own survival during this situation. In Experiment 4, Krause et al. used scenarios that did not make reference to the participant also being included during the imagined task and pitted the third person survival of "your youngest blood relative" to that of "a friend (who is not a member of your family)," and to a famous person, "singer Ariana Grande" in a manner similar to the procedure of Cunningham et al. (2013) who used Prime Minister David Cameron. Unlike Cunningham et al., there was no difference in recall between the friend or the famous person, nor was there a difference in recall between those two conditions and the youngest blood relative. This consistent finding of null 
results may have to do with Krause et al. using a 5-minute distractor task between encoding and test compared to the 2-minute distractor task used in our and many other adaptive memory procedures, or their absence of a difference between kin and non kin may be due to the potential saliency of using children in our scenarios compared to their using adults. Future studies will be necessary to explore the boundary conditions and replicability of this effect.

It should not be surprising that the human memory system differentially encodes different types of information and that biologically relevant information receives privileged treatment. Biological constraints on learning have been widely recognized among animal learning researchers. Thorndike (1911) identified the concept of belongingness in his puzzle box experiments to describe the finding that certain responses appeared to 'belong' in certain situation contexts (i.e., pull string to escape) and thus could be reinforced but responses that did not 'belong' in the context (i.e., grooming to escape) could not be reinforced. Seligman (1970) suggested that an organism comes 'prepared' to more readily associate certain events that are relevant to survival. Fittingly, avoidance of novel tastes is acquired more rapidly when the flavor is paired with gastric distress instead of footshock (Garcia \& Koelling, 1966) and selective associations like this have been observed in animals as young as 1-day old rat pups (Gemberling \& Domjan, 1982). Pavlovian conditioning creates associations between contiguously occurring events, but it has long been known that biologically relevant unconditioned stimuli (food or shock) become associated to a conditioned stimulus more readily than they do to biologically neutral unconditioned stimuli (tone or light) and these biologically significant cues are protected from cue competition effects (Miller \& Matute, 1996). That information processed in terms of its relevancy to one's biological child (surviving in the grasslands or putatively parenting that child) is better remembered than an adopted child thus appears functional given the importance of preserving one's genetic lineage. However, this effect should not influence how we view step-parents' ability to raise offspring that are not biologically their own. Cook, Hodes, and Lang (1986) found that associations to neutral stimuli 
paired with snakes were learned faster than neutral stimuli paired with guns. Guns are clearly a more biologically relevant threat in our current environment than snakes and it would be preposterous to argue that we ought to be more afraid of snakes than guns (i.e., the naturalistic fallacy). Thus, many evolutionary predispositions are not particularly relevant or useful in our current environment. That said, these evolved tunings can be demonstrated in controlled laboratory studies and are informative about the evolution of our cognitive faculties.

One potential limitation of our studies of parent processing and a potential future direction is that we did not assess whether being an actual parent influences the basic parent processing effect and/or the effect of biological relatedness on the $3^{\text {rd }}$ person survival and parenting task. That is, our participants were asked to imagine having parented a baby and, while we did not collect data on this, their being college students suggests the large majority are not currently parents. Thus, it might have been difficult for these participants to truly imagine themselves in this scenario, and the potential motivating features of raising children might have been small. Similarly, physiological changes that accompany the raising of a child, in both men and women, may also impact mnemonic capabilities on a task of this nature. Therefore, it would be of interest in the future to explore whether or not this parent processing effect is enhanced for actual parents compared to nonparents, and to determine whether actual parents would be more affected by the biological relatedness of the child in either task.

The effects reported here are congruent with an adaptive memory framework. Though evolutionary explanations are often criticized as being 'just so stories,' the effects demonstrated in the present studies fit a priori predictions. Instead of observing behavioral patterns and providing a post hoc explanation, our experiments, and those of other adaptive memory researchers, were designed to test predictions predicated on the evolutionary perspective (Nairne et al., 2017). Similarly, the Omega scaling factor proposed by Seitz et al. (in press) provides a conceptual tool for modelling and predicting how memory will proceed based on the perceived fitness relevance of encoded information. The survival processing effect is well 
established in the literature and the adaptive memory framework continues to provide a useful heuristic in understanding how the human memory system operates, which is not to deny that at a different level of analysis these effects might also be explicable in terms of more proximal memory mechanisms. We hope that the present observations will encourage others to continue to explore the importance of biological relevancy influencing mnemonic performance. 


\section{References}

Anderson, J. R., \& Schooler, L. J. (2000). The adaptive nature of memory. In E. Tulving \& F. I. M. Craik (Eds.), The Oxford Handbook of Memory (pp. 557-570). New York, NY: Oxford University Press.

Baker, M. D., Nicole Sloan, H., Hall, A. D., Leo, J., \& Maner, J. K. (2015). Mating and memory: Can mating cues enhance cognitive performance? Evolutionary Psychology, 13(4). https://doi.org/10.1177/1474704915623280

Barkow, J.H., Cosmides, L., \& Tooby, J. (1992). The Adapted Mind. Oxford University Press.

Bell, R., \& Buchner, A. (2012). How adaptive is memory for cheaters? Current Directions in Psychological Science, 21(6), 403-408. https://doi.org/10.1177/0963721412458525

Bonin, P., Thiebaut, G., Witt, A., \& Méot, A. (2019). Contamination Is "good" for your memory! Further evidence for the adaptive view of memory. Evolutionary Psychological Science, 117. https://doi.org/10.1007/s40806-019-00188-y

Bower, G. H., \& Gilligan, S. G. (1979). Remembering information related to one's self. Journal of Research in Personality, 13(4), 420-432. https://doi.org/10.1016/0092-6566(79)90005-9

Cook, E. W., Hodes, R. L., \& Lang, P. J. (1986). Preparedness and phobia: Effects of stimulus content on human visceral conditioning. Journal of Abnormal Psychology, 95(3), 195-207. https://doi.org/10.1037/0021-843X.95.3.195

Craik, F. I. M., \& Lockhart, R. S. (1972). Levels of processing: A framework for memory research. Journal of Verbal Learning and Verbal Behavior, 11(6), 671-684. https://doi.org/10.1016/S0022-5371(72)80001-X

Cunningham, S. J., Brady-Van den Bos, M., Gill, L., \& Turk, D. J. (2013). Survival of the selfish: Contrasting self-referential and survival-based encoding. Consciousness and Cognition, 22(1), 237-244. https://doi.org/10.1016/j.concog.2012.12.005 
Denniston, J. C., Miller, R. R., \& Matute, H. (1996). Biological significance as a determinant of cue competition. Psychological Science, 7(6), 325-331. https://doi.org/10.1111/j.14679280.1996.tb00383.x

Derringer, C. J., Scofield, J. E., \& Kostic, B. (2017). Investigations of a reproductive processing advantage in memory. Memory and Cognition, 45(6), 983-1001. https://doi.org/10.3758/s13421-017-0709-0

Domjan, M., \& Krause, M. (2017). Generality of the laws of learning: From biological constraints to ecological perspectives. In J. H. Byrne (Ed.), Learning and Memory: A Comprehensive Reference (pp. 189-201). https://doi.org/10.1016/b978-0-12-809324-5.21012-2

Faul, F., Erdfelder, E., Lang, A.G., \& Buchner, A. (2007). G*Power 3: a flexible statistical power analysis program for the social, behavioral, and biomedical sciences. Behavior Research Methods, 39(2), 175-191. http://www.ncbi.nlm.nih.gov/pubmed/17695343

Fernandes, N. L., Pandeirada, J. N. S., Soares, S. C., \& Nairne, J. S. (2017). Adaptive memory: The mnemonic value of contamination. Evolution and Human Behavior. 38(4). 451-460. https://doi.org/10.1016/j.evolhumbehav.2017.04.003

Garcia, J., \& Koelling, R. A. (1966). Relation of cue to consequence in avoidance learning. Psychonomic Science, 4(1), 123-124. https://doi.org/10.3758/BF03342209

Gelin, M., Bonin, P., Méot, A., \& Bugaiska, A. (2018). Do animacy effects persist in memory for context? Quarterly Journal of Experimental Psychology, 71(4), 965-974. https://doi.org/10.1080/17470218.2017.1307866

Gemberling, G. A., \& Domjan, M. (1982). Selective associations in one-day-old rats: Tastetoxicosis and texture-shock aversion learning. Journal of Comparative and Physiological Psychology, 96(1), 105-113. https://doi.org/10.1037/h0077855

Gretz, M. R., \& Huff, M. J. (2019). Did you wash your hands? Evaluating memory for objects 
touched by healthy individuals and individuals with contagious and non-contagious diseases. Applied Cognitive Psychology. 1-8. https://doi.org/10.1002/acp.3604

Horgan, T. G., Broadbent, J., McKibbin, W. F., \& Duehring, A. J. (2016). Show versus tell? The effects of mating context on women's memory for a man's physical features and verbal statements. Journal of Social and Personal Relationships, 33(6), 733-750.

https://doi.org/10.1177/0265407515590279

Hou, C., \& Liu, Z. (2019). The survival processing advantage of face: The memorization of the (un)trustworthy face contributes more to survival adaptation. Evolutionary Psychology, 17(2), 1-12. https://doi.org/10.1177/1474704919839726

Kang, S. H. K., McDermott, K. B., \& Cohen, S. M. (2008). The mnemonic advantage of processing fitness-relevant information. Memory and Cognition, 36(6), 1151-1156. https://doi.org/10.3758/MC.36.6.1151

Kazanas, S. A., \& Altarriba, J. (2015). The survival advantage: Underlying mechanisms and extant limitations. Evolutionary Psychology, 13(2), https://doi.org/10.1177/147470491501300204

Klein, S. B. (2013). Does optimal recall performance in the adaptive memory paradigm require the encoding context to encourage thoughts about the environment of evolutionary adaptation? Memory \& Cognition, 41(1), 49-59. https://doi.org/10.3758/s13421-012-0239-8

Kole, J. A., \& Healy, A. F. (2011). Memory for details about people: familiarity, relatedness, and gender congruency. Memory \& Cognition, 39(4), 637-648. https://doi.org/10.3758/s13421010-0051-2

Krause, M. A., Trevino, S., Cripps, A., Chilton, K., Sower, E., \& Taylor, J. P. (2019). Inclusive fitness does not impact the survival processing effect. Animal Behavior and Cognition, 6(1), 13-31. https://doi.org/10.26451/abc.06.01.02.2019 
Miller, R. R., \& Matute, H. (1996). Biological significance in forward and backward blocking: Resolution of a discrepancy between animal conditioning and human causal judgment. Journal of Experimental Psychology: General, 125(4), 370-386.

https://doi.org/10.1037/0096-3445.125.4.370

Nairne, J. S., \& Pandeirada, J. N. S. (2016). Adaptive memory: The evolutionary significance of survival processing. Perspectives on Psychological Science, 11(4), 496-511. https://doi.org/10.1177/1745691616635613

Nairne, J. S., Pandeirada, J. N. S., \& Thompson, S. R. (2008). Adaptive memory: The comparative value of survival processing. Psychological Science, 19(2), 176-180. https://doi.org/10.1111/j.1467-9280.2008.02064.x

Nairne, J. S., Thompson, S. R., \& Pandeirada, J. N. S. (2007). Adaptive memory: Survival processing enhances retention. Journal of Experimental Psychology: Learning, Memory, and Cognition, 33(2), 263-273. https://doi.org/10.1037/0278-7393.33.2.263

Nairne, J. S., VanArsdall, J. E., \& Cogdill, M. (2017). Remembering the living. Current Directions in Psychological Science, 26(1), 22-27. https://doi.org/10.1177/0963721416667711

Nairne, J. S., VanArsdall, J. E., Pandeirada, J. N. S., Cogdill, M., \& LeBreton, J. M. (2013). Remembering the living: Episodic memory Is tuned to animacy. Psychological Science, 24(10), 2099-2105. https://doi.org/10.1177/0956797613480803

New, J., Krasnow, M. M., Truxaw, D., \& Gaulin, S. J.C. (2007). Spatial adaptations for plant foraging: Women excel and calories count. Proceedings of the Royal Society B: Biological Sciences, 274(1626), 2679-2684. https://doi.org/10.1098/rspb.2007.0826

Pandeirada, J. N. S., Fernandes, N. L., Vasconcelos, M., \& Nairne, J. S. (2017). Adaptive memory: Remembering potential mates. Evolutionary Psychology, 15(4), https://doi.org/10.1177/1474704917742807 
Sandry, J., Trafimow, D., Marks, M. J., \& Rice, S. (2013). Adaptive memory: Evaluating alternative forms of fitness-relevant processing in the survival processing paradigm. PLoS ONE, 8(4). https://doi.org/10.1371/journal.pone.0060868

Schaller, M. (2018). The parental care motivational system and why it matters (for everyone). Current Directions in Psychological Science, 27(5), 295-301. https://doi.org/10.1177/0963721418767873

Schaller, M. (2020). Evolutionary psychology meets socio-ecological psychology: the motivational psychologies of disease-avoidance and parental care. Current Opinion in Psychology, 32, 6-11. https://doi.org/10.1016/j.copsyc.2019.06.020

Scofield, J. E., Buchanan, E. M., \& Kostic, B. (2018). A meta-analysis of the survival-processing advantage in memory. Psychonomic Bulletin \& Review, 25(3), 997-1012. https://doi.org/10.3758/s13423-017-1346-0

Seitz, B.M., Blaisdell, A. P., Polack, C. W., \& Miller, R. R. (in press). The role of biological significance in human learning and memory. International Journal of Comparative Psychology.

Seitz, B. M., Polack, C. W., \& Miller, R. R. (2018). Adaptive memory: Is there a reproductionprocessing effect? Journal of Experimental Psychology: Learning, Memory, and Cognition, 44(8), 1167-1179. https://doi.org/10.1037/xIm0000513

Seligman, M. E. (1970). On the generality of the laws of learning. Psychological Review, 77(5), 406-418. https://doi.org/10.1037/h0029790

Shettleworth, S. J. (1972). Constraints on learning. Advances in the Study of Behavior, 4, 1-68. https://doi.org/10.1016/S0065-3454(08)60006-6

Smith, D. S., Jones, B. C., Feinberg, D. R., \& Allan, K. (2012). A modulatory effect of male voice pitch on long-term memory in women: evidence of adaptation for mate choice? Memory \& 
Cognition, 40(1), 135-144. https://doi.org/10.3758/s13421-011-0136-6

Thorndike, E. (1911). Animal intelligence: Experimental studies.

Weinstein, Y., Bugg, J. M., \& Roediger, H. L. (2008). Can the survival recall advantage be explained by basic memory processes? Memory and Cognition, 36(5), 913-919. https://doi.org/10.3758/MC.36.5.913 


\section{Author Notes}

This research was supported in part by NIMH grant 33881. Benjamin Seitz is supported by National Science Foundation Grant DGE-1650604. The authors declare that they have no conflicts of interest. They are grateful to Crystal Casado, Michelle Jeon, and Tori Pena for their efforts in collecting the data. 
Survival Scenario (Experiment 1)

In this task, we would like you to imagine that you are stranded, thousands of years ago, in the grasslands of a foreign land, without any basic survival materials. Over the next few months, you'll need to find steady supplies of food and water and protect yourself from predators. We are going to show you a list of words, and we would like you to rate how relevant each of these words would be for you in this survival situation. Some of the words may be relevant and others may not it's up to you to decide.

\section{Biological Parenting Scenario (Experiment 1, 2, 3)}

In this task, we would like you to imagine that you are living, thousands of years ago, in the grasslands of a foreign land, and have just parented a baby. Over the next few months, you'll need to provide care and nourishment for your baby and help him/her grow. We are going to show you a list of words, and we would like you to rate how relevant each of these words would be for you in this child-rearing situation. Some of the words may be relevant and others may not - it's up to you to decide.

\section{Adopted Parenting Scenario (Experiment 1, 2, 3)}

In this task, we would like you to imagine that you are living, thousands of years ago, in the grasslands of a foreign land, and have just came across a baby that is not yours. Over the next few months, you'll need to provide care and nourishment for this baby that is not yours and help him/her grow. We are going to show you a list of words, and we would like you to rate how relevant each of these words would be for you in this adopted child-rearing situation. Some of the words may be relevant and others may not - it's up to you to decide.

\section{Pet-Raising Scenario (Experiment 1)}

In this task, we would like you to imagine that you are living, thousands of years ago, in the grasslands of a foreign land, and have just domesticated a young, wild dog. Over the next few months, you'll need to provide care and nourishment for this dog and help it grow. We are going to show you a list of words, and we would like you to rate how relevant each of these words would be for you in this pet-raising situation. Some of the words may be relevant and others may not - it's up to you to decide.

\section{Biological Third-Person Survival Scenario (Experiment 2)}

In this task, we would like you to imagine that your baby is stranded, thousands of years ago in the grasslands of a foreign land, without any basic survival materials. Over the next few months, your baby will need to find steady supplies of food and water and protect itself from predators. We are going to show you a list of words, and we would like you to rate how relevant each of these words would be for your baby in this survival situation. Some of the words may be relevant and others may not - it's up to you to decide.

\section{Adopted Third-Person Survival Scenario (Experiment 2)}

In this task, we would like you to imagine that a baby that is not yours is stranded, thousands of years ago in the grasslands of a foreign land, without any basic survival materials. Over the next few months, the baby will need to find steady supplies of food and water and protect itself from predators. We are going to show you a list of words, and we would like you to rate how relevant each of these words would be for this baby that is not yours in this survival situation. Some of the words may be relevant and others may not - it's up to you to decide.

Table 1. Scenario descriptions for all experiments 


\begin{tabular}{lllll}
\hline bath & dinner & grass & pepper & son \\
\hline bed & dirt & home & python & stem \\
\hline bird & disease & horse & river & storm \\
\hline blood & emerald & juice & rod & sugar \\
\hline cat & finger & liver & salt & tobacco \\
\hline child & friend & mountain & silk & valley \\
\hline clothing & fruit & needle & snow & village \\
\hline cotton & fur & orange & sock & water \\
\hline
\end{tabular}

Table 2. Word List for Experiment 1. 


\begin{tabular}{llllll}
\hline ant & spinach & ball & bee & python & hair \\
\hline storm & horn & tree & fruit & water & food \\
\hline potato & pig & guitar & knife & penny & horse \\
\hline fish & grass & shoes & cat & stick & priest \\
\hline hammer & oxygen & sock & rod & clothing & emerald \\
\hline
\end{tabular}

Table 3. Word List for Experiment 2. 


\begin{tabular}{|c|c|c|c|c|c|c|}
\hline Scenario & Arousing & Aversive & Challenging & Familiar & Imaginative & Interesting \\
\hline Survival (Exp. 1) & $2.1+/-1.2$ & $2.8+/-1.2$ & $4.4+/-.96$ & $1.9+/-1.2$ & $3.2+/-1.2$ & $3.6+/-1.1$ \\
\hline Adopted Parenting (Exp.1,2,3) & $2.0+/-1.2$ & $2.7+/-1.0$ & $4.3+/-.94$ & $1.8+/-1.1$ & $2.8+/-1.2$ & $3.5+/-1.0$ \\
\hline Biological Parenting (Exp. 1,2,3) & $1.7+/-.93$ & $2.6+/-1.0$ & $4.1+/-1.1$ & $1.9+/-1.1$ & $2.9+/-1.2$ & $3.1+/-1.0$ \\
\hline Dog Raising (Exp.1) & $2.2+/-1.4$ & $2.3+/-1.0$ & $3.3+/-1.6$ & $2.6+/-1.3$ & $3.5+/-1.2$ & $3.6+/-1.1$ \\
\hline Third-Person Survival Adopted (Exp. 2) & $1.9+/-1.1$ & $2.8+/-1.3$ & $4.2+/-1.3$ & $1.6+/-1.0$ & $2.6+/-1.3$ & $3.4+/-.98$ \\
\hline Third-Person Survival Biological (Exp. 2) & $1.9+/-1.1$ & $3.0+/-1.3$ & $4.5+/-1.0$ & $1.6+/-.91$ & $2.2+/-1.1$ & $3.5+/-1.1$ \\
\hline
\end{tabular}

Table 4. Mean descriptive qualities of each scenario and standard deviation 
a

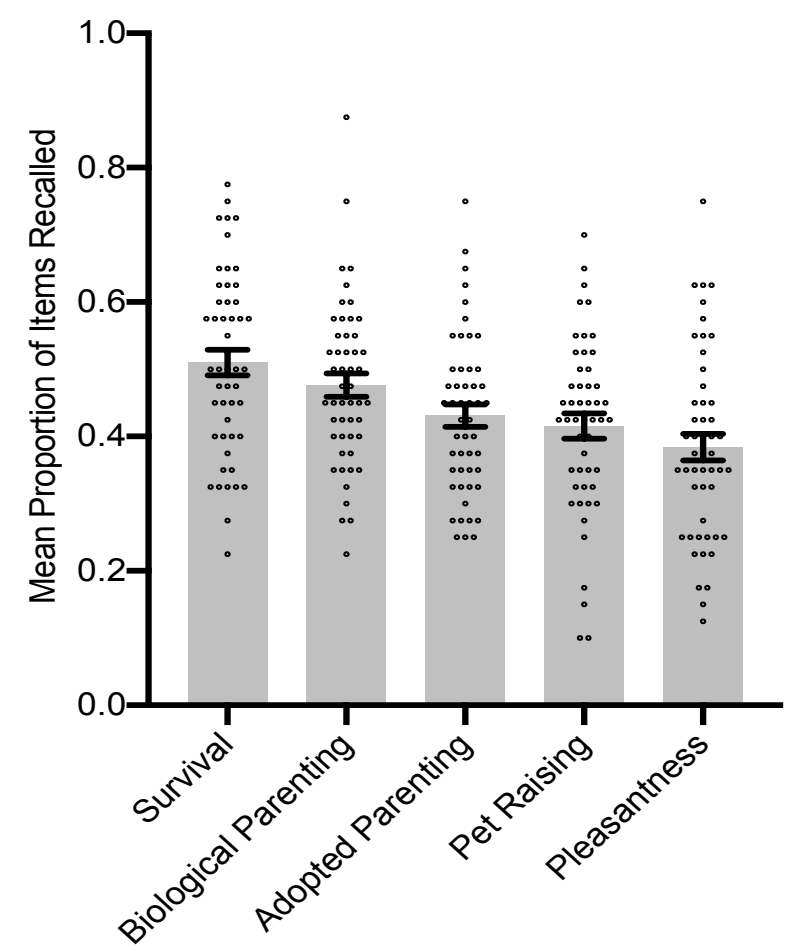

C

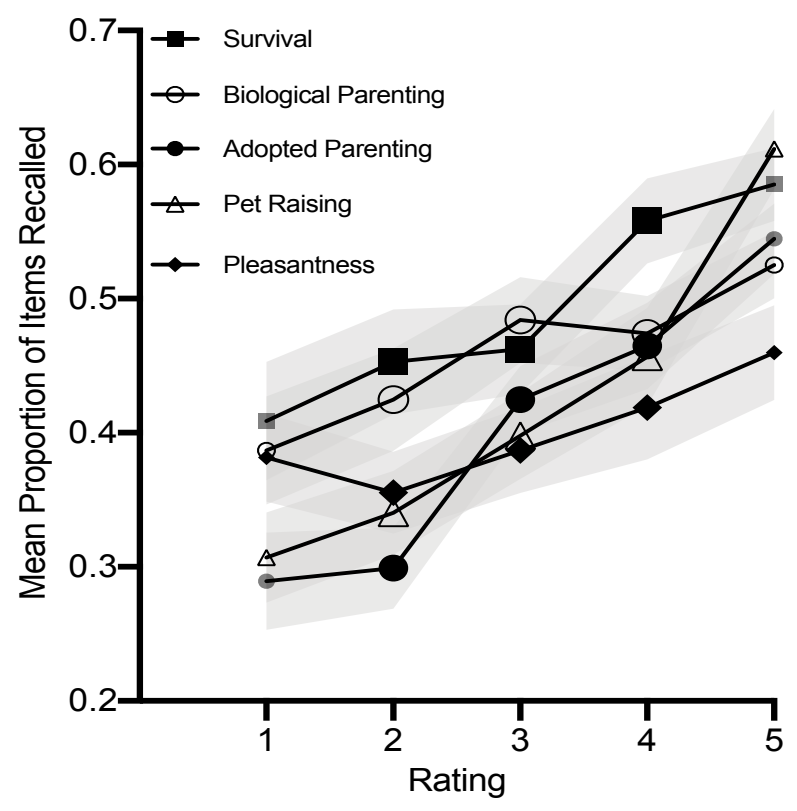

b

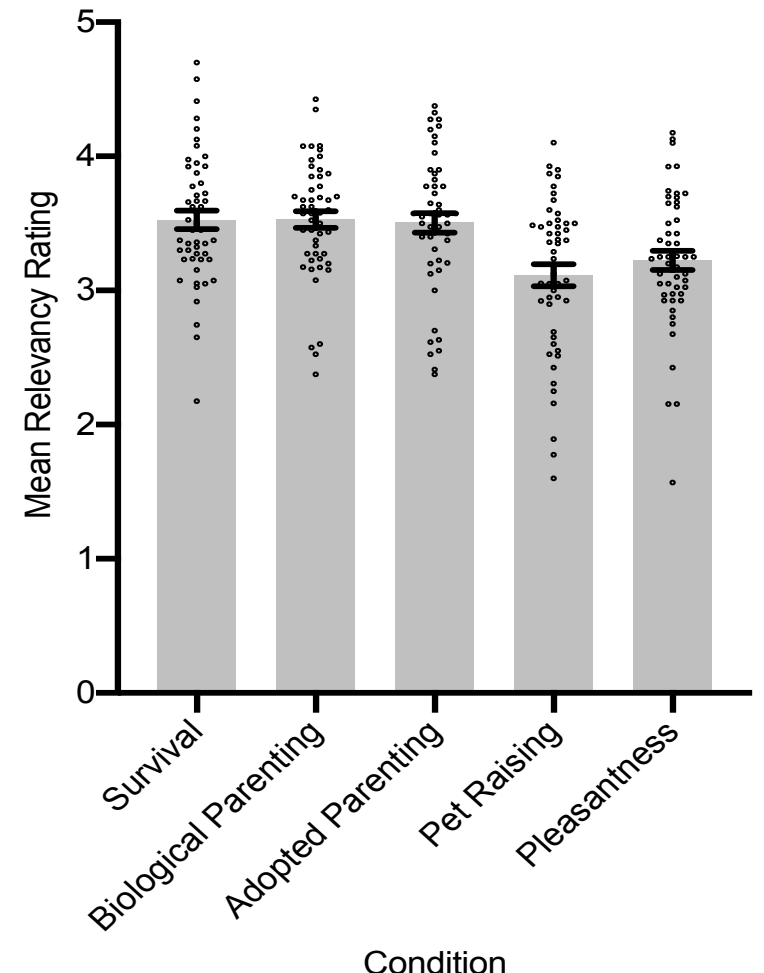

Figure 1: Results from experiment 1. All error bars represent SEM. a) Mean proportion of items (out of 40) recalled as a function of condition. b) Mean relevancy ratings as a function of condition. c) Proportion of words recalled as a function of relevancy rating (1-5 scale). Subjects were randomly assigned to a condition, but item ratings are a within-subjects variable. Recall of items excluding those with extremely low (1) and high (5) relevancy ratings were removed from the final analysis. 
a
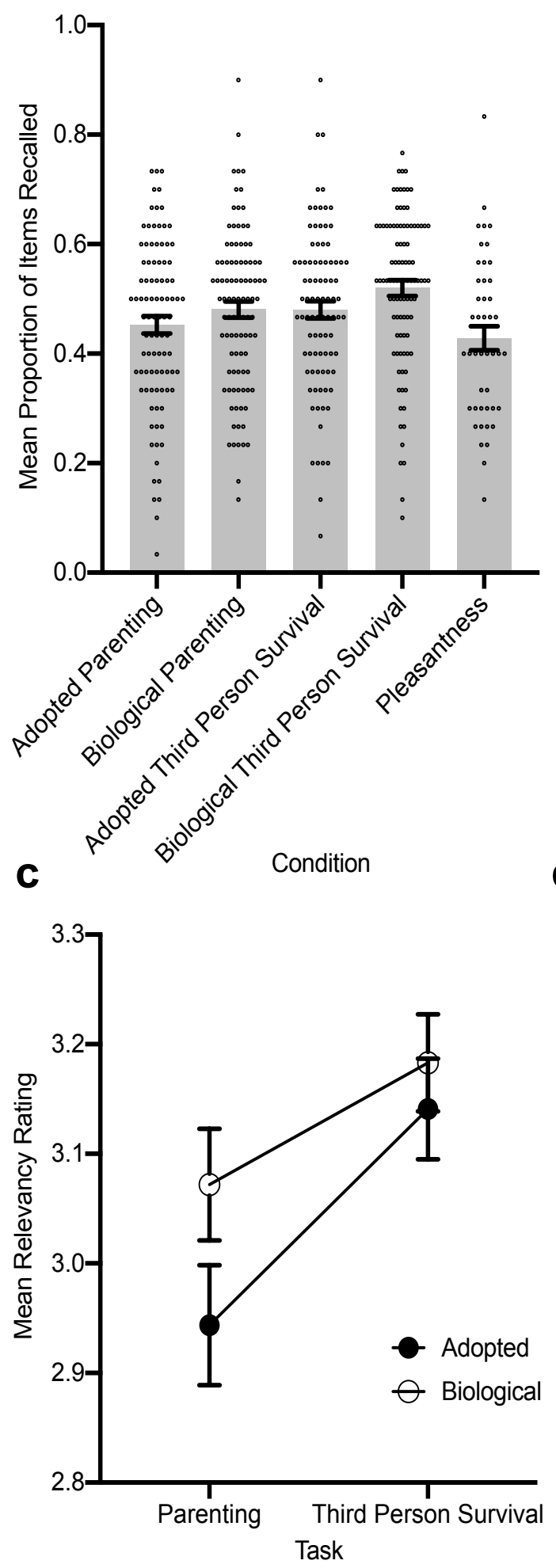

b

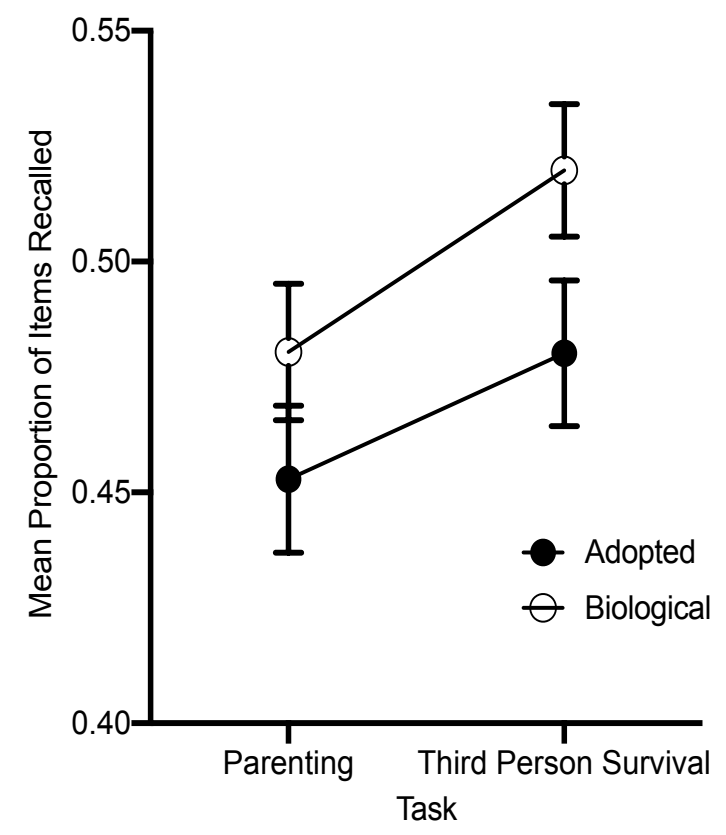

d

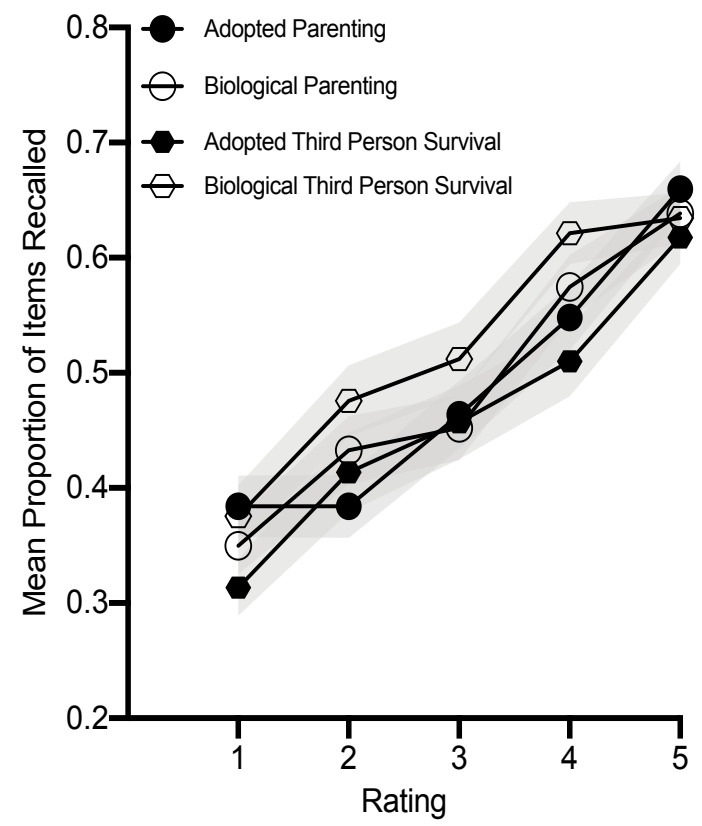

Figure 2: Results from Experiment 2. All error bars represent SEM. a) Mean proportion of items (out of 30) recalled as a function of condition. b) Mean proportion of items (out of 30) recalled as a function of task and relatedness. c) Mean relevancy ratings of items (out of 30) as a function of task and relatedness. Error bars represent standard error of the mean. d) Proportion of words recalled as a function of relevancy rating (1-5 scale). Participants were randomly assigned to a condition, but item ratings are a within-subjects variable. 


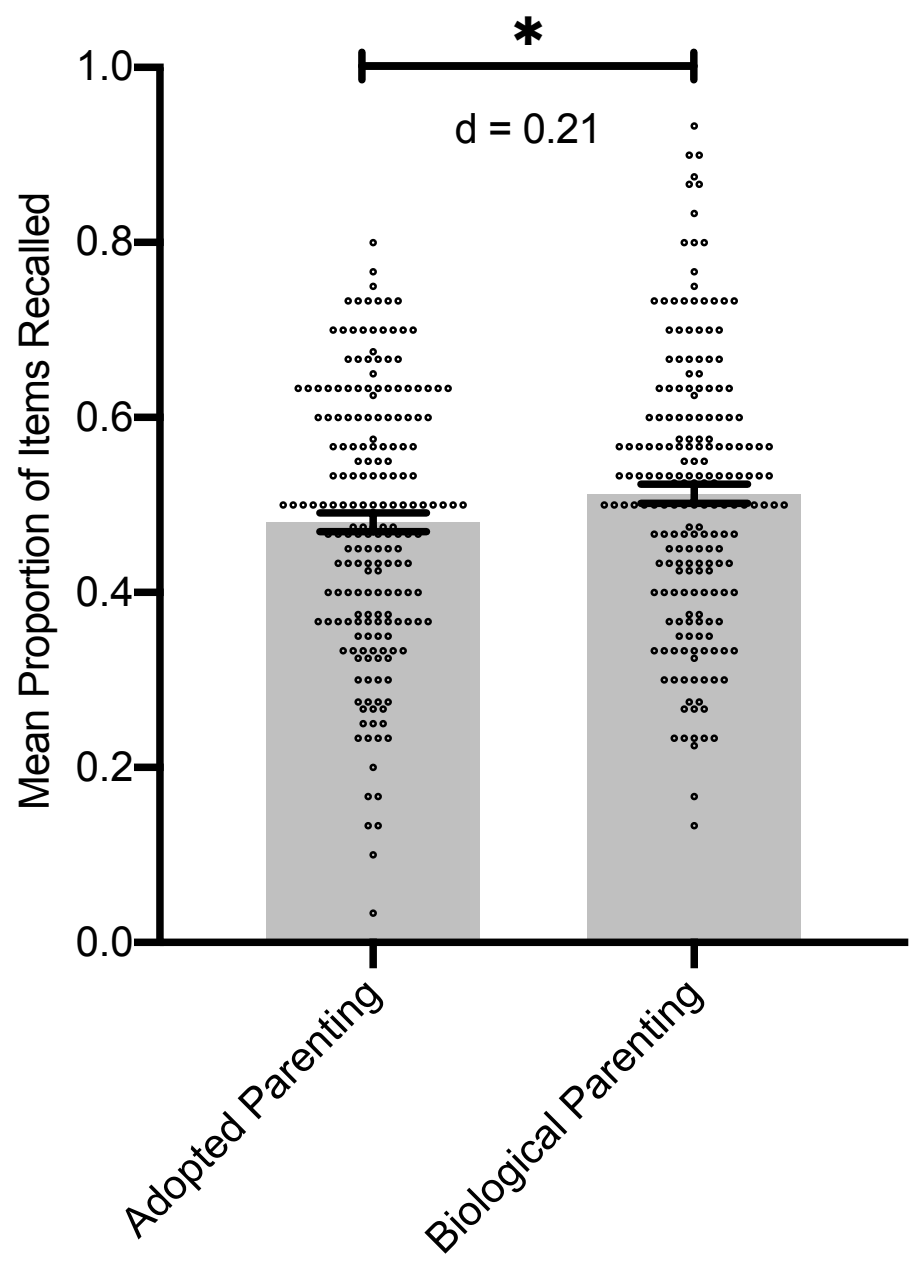

Figure 3. Results from an internal meta-analysis including data from experiments 1-3. Mean word recall as a function of condition. Error bars represent standard error of the mean. $p<.05$. 